\title{
Anti-proliferative Effect of Licochalcone A on Vascular Smooth Muscle Cells
}

\author{
Jin-Hee PARK, ${ }^{a}$ Hyun Joung Lim, ${ }^{a}$ Kuy-Sook LeE, ${ }^{a}$ Seahyoung LeE, ${ }^{a}$ Hyun-Jeong KwaK, ${ }^{a}$ \\ Jeong-Heon $\mathrm{CHA}^{b}$ and Hyun-Young PARK ${ }^{*}, a$ \\ ${ }^{a}$ Division of Cardiovascular and Rare Diseases, Center for Biomedical Sciences, NIH; 194, Tongillo, Eunpyeong-gu, Seoul \\ 122-701, Republic of Korea: and ${ }^{b}$ Department of Oral Biology, BK21 Project, Oral Science Research Center, Yonsei \\ University College of Dentistry; 134 Shinchon-dong, Seodaemun-gu, Seoul 120-749, Republic of Korea. \\ Received April 1, 2008; accepted August 6, 2008; published online August 28, 2008
}

Licochalcone A, a flavonoid found in licorice root (Glycyrrhiza glabra), is known for its anti-microbial activity and its reported ability to inhibit cancer cell proliferation. In the present study, we investigated whether licochalcone A inhibits rat vascular smooth muscle cell (rVSMC) proliferation. Our data indicate that $5 \mu \mathrm{M}$ licochalcone A inhibited platelet-derived growth factor (PDGF)-induced rVSMC proliferation, possibly through its ability to block the progression of the cell cycle from $G_{1}$ to $S$ phase. In addition, $5 \mu_{M}$ licochalcone $A$ significantly inhibited the PDGF-induced expression of cyclin A, cyclin D1, CDK2, and CDK4, and the phosphorylation of Rb. Licochalcone A also reversed the decrease in p2 $7^{\mathrm{kip} 1}$ expression reduced by PDGF. Finally, licochalcone A inhibited the PDGF-induced activation of extracellular signal-regulated kinase (ERK)1/2. Together, these data provide the first evidence that licochalcone A can regulate rVSMC proliferation and suggest that licochalcone $A$ inhibits the proliferation of rVSMCs by suppressing the PDGF-induced activation of the ERK1/2 pathway and $R b$ phosphorylation, resulting in cell cycle arrest.

Key words licochalcone A; cellular proliferation; cell cycle; smooth muscle cell

Vascular smooth muscle cell (VSMC) proliferation is a key step in the development of pathogenic vascular conditions such as restenosis and atherosclerosis. ${ }^{1)}$ Therefore, the identification of a novel agent that can inhibit VSMC proliferation and the elucidation of its underlying mechanism will aid in the development of therapeutic strategies aimed at preventing such pathologic vascular conditions. Licochalcone A, a flavonoid found in licorice root (Glycyrrhiza glabra), possesses multiple biological properties, including anti-microbial, anti-Legionella, anti-inflammatory, and anti-spasmodic effects. ${ }^{2)}$ Licochalcone A has been used to treat various abdominal spasmodic symptoms in Japan. ${ }^{3)}$ Recent studies have shown that licochalcone $\mathrm{A}$ also has an anti-cancer effect and induces apoptosis in MCF-7 and HL-60 cells. ${ }^{4)}$ These findings suggest that licochalcone A may be a potent inhibitor of rat VSMC (rVSMC) proliferation.

PDGF induces VSMC proliferation by triggering the activation of both the extracellular signal-regulated kinase (ERK)1/2 mitogen-activated protein kinase (MAPK) and phosphatidylinositol (PI) 3-kinase/Akt pathways. The importance of these signaling pathways in PDGF-induced cellular proliferation and DNA synthesis was demonstrated previously. ${ }^{5)}$ Furthermore, activation of the ERK1/2 pathway is essential for cell cycle progression in VSMCs in the presence of mitogens or serum. ${ }^{6}$

Little is known regarding the role of licochalcone $\mathrm{A}$ in VSMC proliferation; thus, we examined its effect on PDGFinduced rVSMC proliferation and the underlying mechanisms. We specifically focused on its effect on the ERK1/2 pathway and cell cycle-related molecules, including $\mathrm{p} 27^{\mathrm{kip} 1}$, cyclin A, cyclin D1, cyclin E, CDK2, and CDK4. Our data indicate that licochalcone $\mathrm{A}$ inhibits the PDGF-induced proliferation of rVSMCs. This anti-proliferative effect appears to stem from the ability of licochalcone A to suppress ERK1/2 and subsequent Elk-1 activation, which in turn, inhibits the expression of cell cycle regulators such as cyclin
A, cyclin D1, CDK2, CDK4 complex, and Rb.

To the best of our knowledge, this study is the first to show that licochalcone A has a potent regulatory effect on pathological vascular conditions such as restenosis. The results of this study will clarify and expand our understanding of the pharmacological activity of licochalcone A in the cardiovascular system.

\section{MATERIALS AND METHODS}

Cell Culture and Reagents Purified licochalcone A was purchased from Calbiochem (San Diego, CA, U.S.A.). rVSMCs were isolated from the thoracic aorta of SpragueDawley rats (200-250 g; ORIENT-Charles River Technology, Seoul, Korea). The cells were maintained in DMEM containing $10 \%$ fetal bovine serum. When the cells reached about $70 \%$ confluency, they were starved in DMEM containing $0.1 \%$ fetal bovine serum for $24 \mathrm{~h}$. After starvation, the cells were pretreated with $5 \mu \mathrm{M}$ licochalcone A for $1 \mathrm{~h}$. Cellular proliferation was induced by adding $10 \mathrm{ng} / \mathrm{ml} \mathrm{PDGF}$ to the medium.

Cytotoxicity Assay The cells were seeded in 48-well plates $\left(2 \times 10^{4}\right.$ cells/well $)$ and incubated with various concentrations of licochalcone A for $24 \mathrm{~h}$. Cytotoxicity was measured using a thiazolyl blue tetrazolium bromide (MTT) colorimetric assay according to the manufacturer's recommendations (Sigma, St. Louis, MO, U.S.A.). The absorbance at $570 \mathrm{~nm}$ was recorded using a microplate reader with SOFTmax PRO software (Molecular Devices).

BrdU Incorporation Assay Cellular proliferation was measured by a BrdU incorporation assay using a commercial ELISA kit according to the manufacturer's instructions (Roche, Basel, Switzerland). Briefly, PDGF-treated cells with or without licochalcone A pretreatment were incubated with BrdU-specific monoclonal antibodies, followed by a peroxidase-conjugated secondary antibody and substrates for 
A

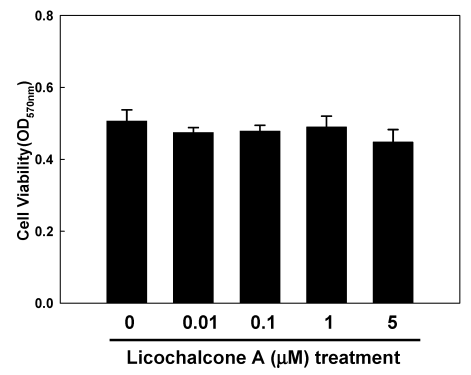

B

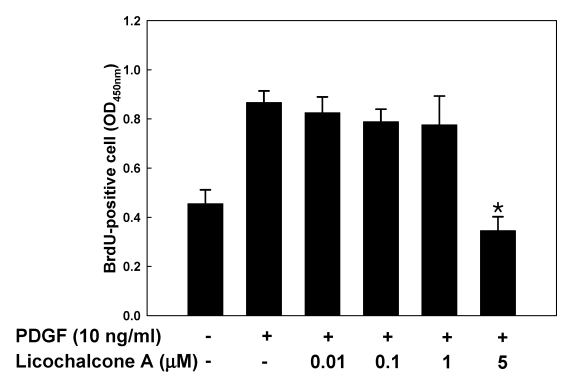

Fig. 1. Licochalcone A Inhibits Rat VSMC Proliferation

(A) Cell viability was measured by MTT assay. (B) Cellular proliferation was measured by BrdU assay. Rat VSMCs were treated with 10 ng/ml PDGF and licochalcone A as indicated. The results are expressed as means \pm S.E.M. Asterisks indicate statistically significant differences $(p<0.05)$.

detection. BrdU incorporation was determined by measuring the absorbance at $450 \mathrm{~nm}$.

Flow Cytometric Analysis The cell cycle distribution was examined by flow cytometry. Synchronized rVSMCs were treated with $5 \mu \mathrm{m}$ licochalcone A for $1 \mathrm{~h}$ prior to the addition of $10 \mathrm{ng} / \mathrm{ml}$ PDGF. The cells were harvested $16 \mathrm{~h}$ after the addition of PDGF and washed with PBS. The cells were then incubated with a buffer containing $0.1 \%$ Triton $\mathrm{X}-100$ and $0.1 \%$ tri-sodium citrate for $30 \mathrm{~min}$, rinsed with PBS, and stained with $50 \mu \mathrm{g} / \mathrm{ml}$ propidium iodide for $20 \mathrm{~min}$ at room temperature. A total of $1 \times 10^{4}$ cells were analyzed with FACScan (Becton Dickson, Franklin Lakes, NJ, U.S.A.).

Western Blot Analysis PDGF-treated cells pretreated with or without $5 \mu \mathrm{M}$ licochalcone A were washed with icecold PBS and lysed in $1 \times$ protein lysis buffer (Cell Signaling, Danvers, MA, U.S.A.). Protein extracts were prepared, separated by SDS-PAGE, and transferred to polyvinylidene fluoride (PVDF) membranes. The blots were incubated with the appropriate primary antibodies. Immunopositive bands were visualized by ECL (Amersham Biosciences, Buckinghamshire, U.K.). $\beta$-Actin was used to correct for loading differences; relative protein expression was determined by densitometric analysis.

Statistics The data are presented as means \pm S.E.M. All statistical analyses were performed using Student's $t$-test. A $p$-value $<0.05$ was considered to indicate statistical significance.

\section{RESULTS}

Cytotoxicity and the Anti-proliferative Effect of Licochalcone A To examine the cytotoxicity of licochalcone A, an MTT assay was performed. No significant cytotoxicity was observed up to $5 \mu \mathrm{m}$ licochalcone A after $24 \mathrm{~h}$ of treatment (Fig. 1A). To investigate the effect of licochalcone A on PDGF-induced rVSMC proliferation, BrdU incorporation was measured. DNA synthesis was significantly increased by PDGF treatment, and the increase was inhibited by pretreatment with licochalcone A (Fig. 1B).

Effect of Licochalcone A on Cell Cycle Progression To further examine the underlying mechanisms of the antiproliferative effect of licochalcone $\mathrm{A}$, we performed a cell cycle analysis using FACS. Cells treated with PDGF for $16 \mathrm{~h}$ exhibited a significant $\mathrm{S}$ phase transition, compared with control cells. Pretreatment with licochalcone A significantly suppressed the PDGF-induced S phase transition (Fig. 2).
A
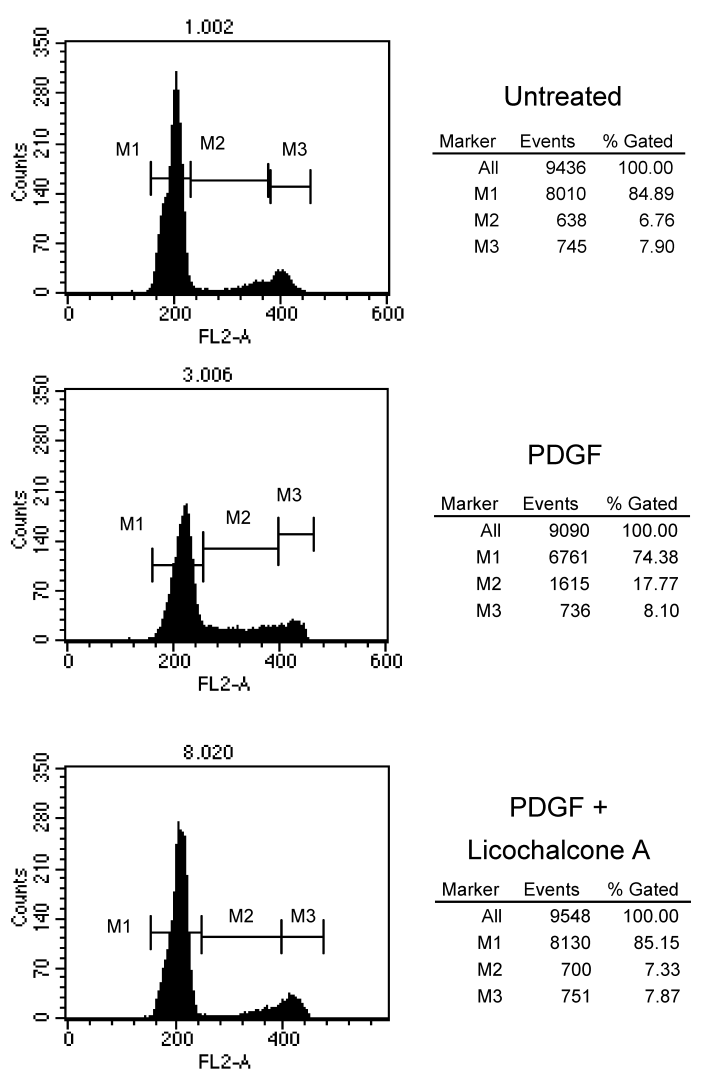

PDGF +

Licochalcone A

\begin{tabular}{rrr} 
Marker & Events & \% Gated \\
\hline All & 9548 & 100.00 \\
M1 & 8130 & 85.15 \\
M2 & 700 & 7.33 \\
M3 & 751 & 7.87
\end{tabular}

B

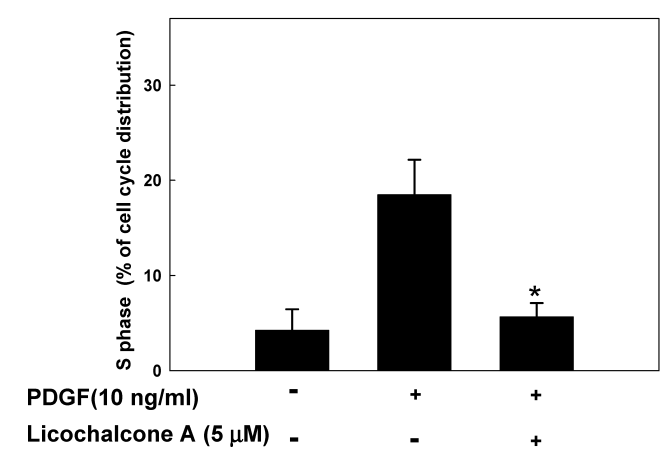

Fig. 2. Licochalcone A Inhibits the PDGF-Induced S Phase Transition

(A) Cell cycle profiles of PDGF-treated rat VSMCs pretreated with $5 \mu \mathrm{M}$ licochalcone A. After $16 \mathrm{~h}$, the cells were harvested and incubated with propidium iodide. The cell cycle distribution was determined by FACS analysis. M1, M2, and M3 in the left panel indicate the percentages of $\mathrm{G}_{0} / \mathrm{G}_{1}, S$, and $\mathrm{G}_{2} / \mathrm{M}$ phase cells, respectively. (B) Comparison of the percentage of cells in $\mathrm{S}$ phase. The results are expressed as means \pm S.E.M. Asterisks indicate statistically significant differences $(p<0.05)$. 
A
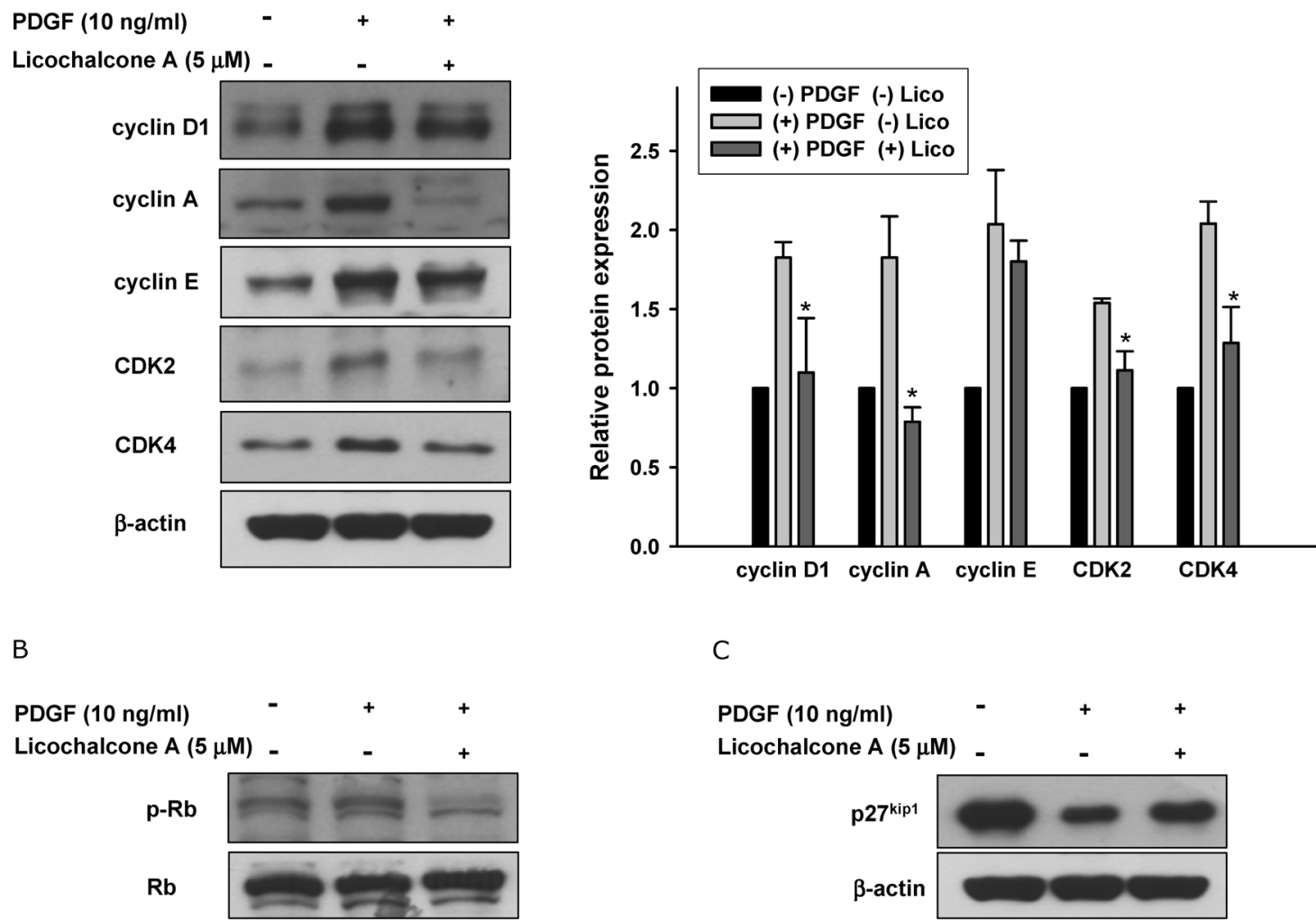

C
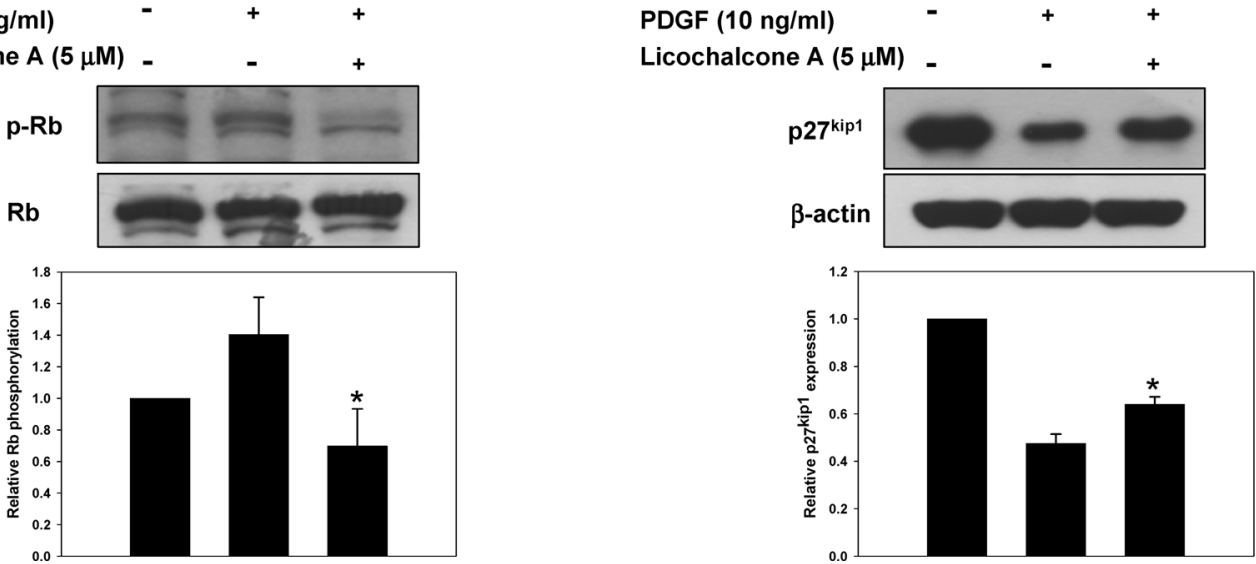

Fig. 3. Effect of Licochalcone A on Cell Cycle Regulatory Protein Expression

(A) Western blot showing cyclin D1, cyclin A, cyclin E, CDK2, and CDK4 expression. The cells were treated with PDGF and licochalcone A as indicated. Relative cyclin/CDK complex expression was quantified. (B) Western blot showing p-Rb and Rb expression. (C) Western blot showing $\mathrm{p} 27^{\mathrm{kip} 1}$ expression. The results are expressed as means \pm S.E.M Asterisks indicate statistically significant differences $(p<0.05)$.

Effect of Licochalcone $A$ on the Expression of Cell Cycle Regulatory Proteins To determine the molecular basis for the cell cycle arrest induced by licochalcone A, we examined the expression of multiple cell cycle regulatory proteins by Western blotting. PDGF significantly increased the expression of cyclin D1, cyclin A, cyclin E, CDK2, and CDK4. Pretreatment with $5 \mu \mathrm{m}$ licochalcone A suppressed the PDGF-induced expression of each of the proteins, except cyclin E (Fig. 3A). The inhibitory effect of licochalcone A on cyclin/CDK complex expression was consistent with the reduced $\mathrm{S}$ phase progression. Compared with control cells, cells treated with PDGF showed significantly increased phosphorylation of $\mathrm{Rb}$, which plays an important role in cell cycle progression. This phosphorylation was significantly suppressed by pretreatment with $5 \mu \mathrm{m}$ licochalcone A (Fig. 3B). We also examined the effect of licochalcone A on the expression of the cell cycle inhibitor $\mathrm{p} 27^{\mathrm{kip} 1}$. The decreased expression of $\mathrm{p} 27^{\mathrm{kip} 1}$ in response to PDGF was reversed by pretreatment with licochalcone A (Fig. 3C). These findings suggest that licochalcone A negatively affects cell cycle progression in PDGF-activated rVSMCs.
Effect of Licochalcone A on Proliferative Signaling Pathways The activation of the ERK1/2 and Akt pathways is important for growth factor-induced VSMC proliferation; therefore, we investigated the effect of licochalcone A on the phosphorylation of ERK1/2 and Akt. PDGF-induced ERK1/2 phosphorylation was significantly diminished by pretreatment with $5 \mu \mathrm{M}$ licochalcone A, whereas Akt phosphorylation was not (Fig. 4A). These results indicate that licochalcone A pretreatment specifically inhibited the ERK1/2 pathway under our experimental conditions. We next examined the phosphorylation of Elk-1, a transcription factor that functions downstream of ERK1/2. The PDGF-increased phosphorylation of Elk-1 was significantly inhibited by licochalcone A pretreatment (Fig. 4B). Together, these results suggest that licochalcone A preferentially inhibits PDGFinduced ERK1/2 phosphorylation and the subsequent phosphorylation of Elk-1.

\section{DISCUSSION}

The herbal extract licochalcone A has been used to treat 


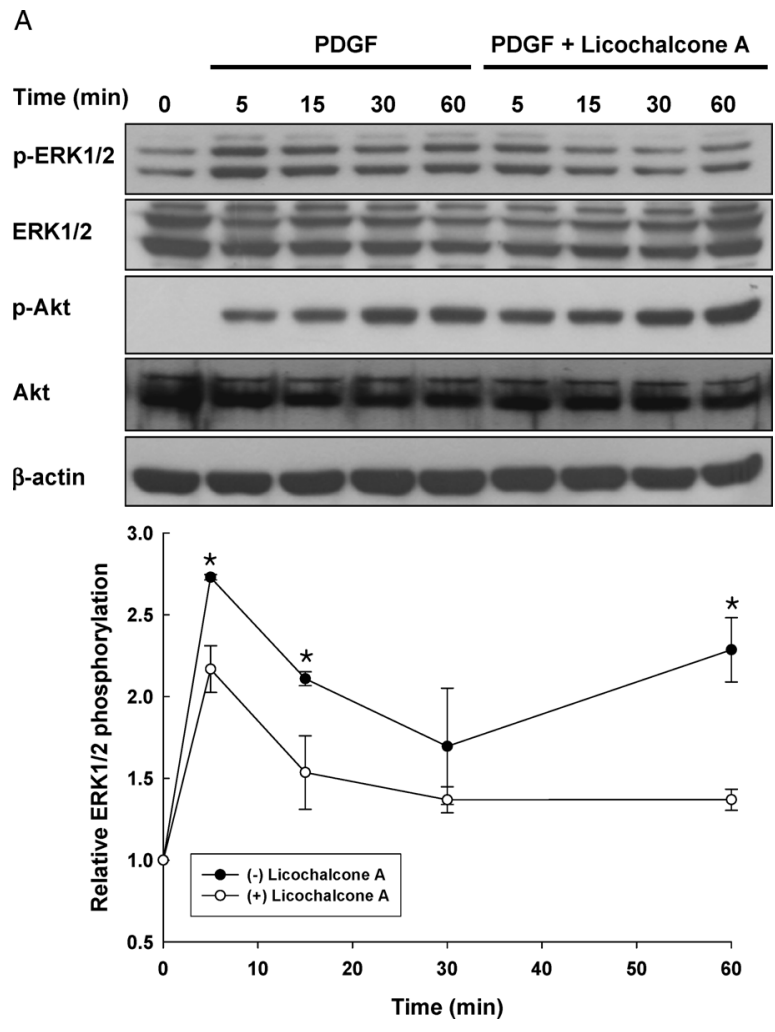

B
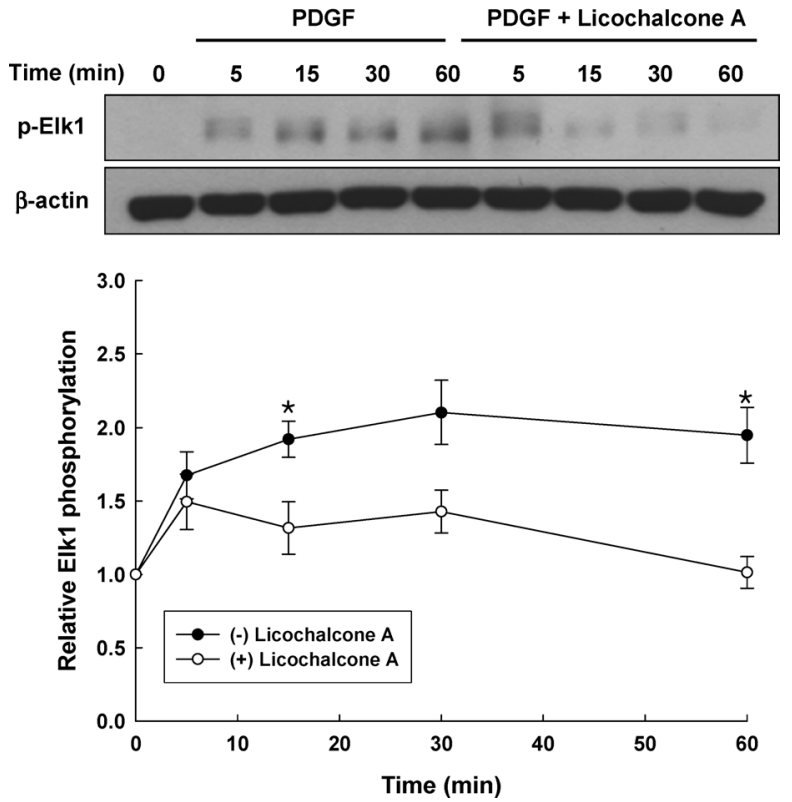

Fig. 4. Licochalcone A Inhibits the PDGF-Induced Phosphorylation of ERK1/2 and Elk-1

(A) Western blot showing p-ERK1/2 and p-Akt expression. The cells were treated with PDGF and licochalcone A as indicated. Relative p-ERK1/2 expression was quantified. (B) Elk-1 phosphorylation was visualized by Western blotting and quantified. The results are expressed as means \pm S.E.M.

infectious Legionella and bacteria, ${ }^{2)}$ and recent studies have indicated that it has additional pharmacological effects. For example, licochalcone $\mathrm{A}$ has been reported to exert a protective effect against ischemia/reperfusion injury, ${ }^{7)}$ to improve renal function, ${ }^{8)}$ and to suppress tumorigenic activity in a variety of cancer cell lines. ${ }^{9,10)}$ Nevertheless, the effect or pharmacological activity of licochalcone A in VSMCs remains elusive. In the present study, we examined its effect on rVSMC proliferation by focusing on cell cycle regulation.

VSMC proliferation is frequently observed in many pathologic vascular conditions such as restenosis and atherosclerosis. Consequently, blocking the proliferation of VSMCs has been a major therapeutic approach in the control of such pathologic conditions. To date, several herbal extracts, including berberine and the flavonoid derivative quercetin, have been reported to inhibit serum- or mitogen-induced VSMC proliferation. ${ }^{11,12)}$ These compounds are known to block the cell cycle in $G_{1}$ phase and decrease cell cycle regulatory protein expression in VSMCs. In the present study, we found that another herbal compound, licochalcone A, significantly inhibits the PDGF-induced proliferation of rVSMCs.

According to our FACS data, $5 \mu \mathrm{m}$ licochalcone A decreased the PDGF-induced transition to $\mathrm{S}$ phase (Fig. 2B). The move from $G_{1}$ to $S$ phase is closely linked to the activation of cell cycle regulatory proteins such as CDKs. In particular, the cyclin D1/CDK4 and cyclin E/CDK2 complexes are essential for entering $\mathrm{S}$ phase. ${ }^{13)} \mathrm{Cyclin} / \mathrm{CDK}$ complexes mediate $\mathrm{Rb}$ phosphorylation in late $\mathrm{G}_{1}$ phase, and hyperphosphorylated $\mathrm{Rb}$ induces the release of the transcription factor E2F, resulting in cell cycle progression to S phase. ${ }^{14,15)}$ In addition, the induction of cyclin A expression, which is regulated by $\mathrm{Rb}$ phosphorylation during late $\mathrm{G}_{1}$ phase, also causes cells to enter S phase. ${ }^{11)}$ On the other hand, the universal CDK inhibitor $\mathrm{p} 27^{\mathrm{kip} 1}$ negatively regulates mitogen-induced cellular proliferation and DNA synthesis. ${ }^{12)}$ Our data also show that licochalcone A decreased the expression of cyclin D1 and CDK4 and the subsequent phosphorylation of $\mathrm{Rb}$, whereas it increased the expression of $\mathrm{p} 27^{\mathrm{kip} 1}$. Thus, both the down-regulation of positive regulators and the up-regulation of negative regulators such as $\mathrm{p} 27^{\mathrm{kip} 1}$ contribute to the cell cycle blocking effect of licochalcone A.

Cell cycle modulators such as cyclin D1 and $\mathrm{p} 27^{\mathrm{kip} 1}$ are regulated by the ERK1/2 pathway. ${ }^{16,17)}$ ERK1/2 signaling plays an important role in PDGF- or serum-induced VSMC proliferation $^{13)}$ as well as hyperplasia formation in vivo. ${ }^{18)}$ Upon mitogenic stimulation, ERK $1 / 2$ is phosphorylated by the kinase MEK1/2. Phosphorylated ERK1/2 then enters the nucleus, where it phosphorylates several downstream transcription factors, including Elk-1. ${ }^{19)}$ The Elk-1 pathway is known to regulate cyclin D1 expression and DNA synthesis in VSMCs. ${ }^{20}$ Licochalcone A significantly decreased the PDGF-induced phosphorylation of ERK1/2 and the subsequent phosphorylation of Elk-1 in our study. This suggests that licochalcone A pretreatment blocks ERK1/2-Elk-1 signaling, which in turn, disrupts the expression of cell cycle regulators and results in cell cycle arrest.

In conclusion, our findings provide the first evidence that licochalcone A has an inhibitory effect on rVSMC proliferation. Our study suggests that licochalcone A is a potent regulator of pathological vascular conditions such as restenosis. Our results will enable us to better understand and re-evaluate the pharmacological activity of licochalcone $\mathrm{A}$ in the cardiovascular system.

Acknowledgments This work was supported by a Korea National Institute of Health intramural research grant (48004845-300-210). 


\section{REFERENCES}

1) Millette E., Rauch B. H., Kenagy R. D., Daum G., Clowes A. W., Trends Cardiovasc. Med., 16, 25-28 (2006).

2) Friis-Moller A., Chen M., Fuursted K., Christensen S. B., Kharazmi A., Planta Med., 68, 416-419 (2002).

3) Nagai H., He J. X., Tani T., Takao T., J. Pharm. Pharmacol., 59, $1421-1426$ (2007).

4) Rafi M. M., Rosen R. T., Vassil A., Ho C. T., Zhang H., Ghai G., Lambert G., DiPaola R. S., Anticancer Res., 20, 2653-2658 (2000).

5) Choudhury G. G., Mahimainathan L., Das F., Venkatesan B., GhoshChoudhury N., Cell Signal, 18, 1854-1864 (2006).

6) Shukla N., Rowe D., Hinton J., Angelini G. D., Jeremy J. Y., Eur. J. Pharmacol., 509, 21-30 (2005).

7) Hwang I. K., Lim S. S., Choi K. H., Yoo K. Y., Shin H. K., Kim E. J., Yoon-Park J. H., Kang T. C., Kim Y. S., Kwon D. Y., Kim D. W., Moon W. K., Won M. H., Acta Pharmacol. Sin., 27, 959-965 (2006).

8) Yokozawa T., Liu Z. W., Chen C. P., Phytomedicine, 6, 439-445 (2000).

9) Jo E. H., Hong H. D., Ahn N. C., Jung J. W., Yang S. R., Park J. S., Kim S. H., Lee Y. S., Kang K. S., J. Agric. Food Chem., 52, 17151719 (2004).

10) Rafi M. M., Vastano B. C., Zhu N., Ho C. T., Ghai G., Rosen R. T.,
Gallo M. A., DiPaola R. S., J. Agric. Food Chem., 50, 677-684 (2002).

11) Fasciano S., Patel R. C., Handy I., Patel C. V., J. Biol. Chem., 280, $15682-15689$ (2005).

12) Fiaschi-Taesch N., Sicari B. M., Ubriani K., Bigatel T., Takane K. K., Cozar-Castellano I., Bisello A., Law B., Stewart A. F., Circ. Res., 99, 933-942 (2006).

13) Wei G. L., Krasinski K., Kearney M., Isner J. M., Walsh K., Andres V., Circ. Res., 80, 418-426 (1997).

14) Zhan Y., Kim O. S., Yasumoto H., Namba M., Miyazaki H., Iwao H., Arterioscler. Thromb. Vasc. Biol., 22, 82-88 (2002)

15) Nie L., Wise M., Peterson D., Meydani M., Free Radic. Biol. Med., 41, $702-708$ (2006).

16) Lavoie J. N., L'Allemain G., Brunet A., Muller R., Pouyssegur J., J. Biol. Chem., 271, 20608-20616 (1996).

17) Castro C., Diez-Juan A., Cortes M. J., Andres V., J. Biol. Chem., 278 4482-4490 (2003)

18) $\mathrm{Hu}$ Y., Cheng L., Hochleitner B. W., Xu Q., Arterioscler. Thromb. Vasc. Biol., 17, 2808-2816 (1997).

19) Yang Z., Madinova A., Kozai T., Joch H., Aebi U., Luscher T. F., Cardiovasc. Res., 53, 227-231 (2002).

20) Adam R. M., Borer J. G., Williams J., Eastham J. A., Loughlin K. R., Freeman M. R., Endocrinology, 140, 5866-5875 (1999). 